\title{
Brain-wide TVA compensation allows rabies virus to retrograde target cell-type-specific projection neurons
}

\author{
Zengpeng Han ${ }^{1,2,3,4 \dagger}$, Nengsong Luo ${ }^{1,3,4,5 \dagger}$, Jiaxin $\mathrm{Kou}^{1,6}$, Lei Li ${ }^{1}$, Zihong $\mathrm{Xu}^{7}$, Siyuan Wei ${ }^{8}$, Yang Wu' , Jie Wang ${ }^{1,2}$, \\ Chaohui $\mathrm{Ye}^{1,2}$, Kunzhang $\operatorname{Lin}^{3,4^{*}}$ (1) and Fuqiang $\mathrm{Xu}^{1,2,3,4,5,9,10^{*}}$
}

\begin{abstract}
Retrograde tracers based on viral vectors are powerful tools for the imaging and manipulation of upstream neural networks projecting to a specific brain region, and they play important roles in structural and functional studies of neural circuits. However, currently reported retrograde viral tracers have many limitations, such as brain area selectivity or the inability to retrograde label genetically defined brain-wide projection neurons. To overcome these limitations, a new retrograde tracing method, AAV-PHP.eB assisted retrograde tracing systems (PARTS) based on rabies virus, was established through brain-wide TVA-dependent targeting using an AAV-PHP.eB that efficiently crosses the bloodbrain barrier in C57BL/6 J mice, and complementation of EnvA-pseudotyped defective rabies virus that specifically recognizes the TVA receptor. Furthermore, combined with Cre transgenic mice, cell-type-specific PARTS (CPARTS) was developed, which can retrograde label genetically defined brain-wide projection neurons. Our research provides new tools and technical support for the analysis of neural circuits.
\end{abstract}

Keywords: Retrograde tracing, Rabies virus, AAV-PHP.eB, Cell-type-specific neurons, Projection neurons

\section{Introduction}

The neural network, which is formed by a large number of neurons of different types connected through synapses, is the structural basis of the brain's complex activities. Understanding the connections between neurons in the nervous system is the challenge of current

\footnotetext{
*Correspondence: kz.lin@siat.ac.cn; fq.xu@siat.ac.cn

${ }^{\dagger}$ Zengpeng Han and Nengsong Luo contributed equally to this work

${ }^{1}$ Key Laboratory of Magnetic Resonance in Biological Systems, State

Key Laboratory of Magnetic Resonance and Atomic and Molecular

Physics, National Center for Magnetic Resonance in Wuhan, Wuhan

Institute of Physics and Mathematics, Innovation Academy for Precision

Measurement Science and Technology, Chinese Academy of Sciences-

Wuhan National Laboratory for Optoelectronics, Wuhan 430071, People's

Republic of China

${ }^{3}$ The Brain Cognition and Brain Disease Institute (BCBDI), Shenzhen

Key Laboratory of Viral Vectors for Biomedicine, Shenzhen

Institute of Advanced Technology, Chinese Academy of Sciences,

Shenzhen 518055, People's Republic of China

Full list of author information is available at the end of the article
}

neuroscience research. Retrograde viral tracers can be used to map and manipulate the upstream neural network projecting to specific brain regions, making them the most promising potential tools for dissecting the structural and functional connections of neural circuits $[1,2]$. Currently, the most commonly used retrograde viral tracers are engineered rabies virus (RV) and adenoassociated virus (AAV), such as N2cG-pseudotyped defective rabies virus (N2cG-RV- $\Delta \mathrm{G}$ ) [3], AAV2-retro [1] and AAV9-retro [4]. Among them, RV, as one of the earliest reported viral tracers due to its unique properties of axon terminal absorption and rapid gene expression, has been used for targeting long-projecting neuronal circuit assemblies [5-8]. AAV2-retro can be used not only to express fluorescent probes to analyse the structural connections of neural networks but also to express functional probes to monitor and manipulate neuronal activities [1]. However, AAV2-retro has brain region selectivity, mainly 
infecting cortical neurons, and N2cG-RV- $\Delta \mathrm{G}$ can only broad-spectrum retrograde infect projection networks that are not cell-type-specific [3]. Therefore, there is an urgent need to establish new retrograde labelling methods for the analysis of genetically defined brain-wide projection neurons received by specific brain regions.

$\mathrm{RV}-\Delta \mathrm{G}$ pseudotyped with the avian sarcoma leucosis virus glycoprotein EnvA can specifically recognize and infect neurons that express the TVA receptor, an avian receptor protein that is absent in mammalian cells [9]. TVA can be expressed by transgenic animals or viral vectors, and retrograde infection of the EnvA-pseudotyped virus along the axon terminal can be restricted to directly input neurons $[10,11]$, but the preparation cycle of transgenic animals is long, and the cost is high. By combining transgenic animals and Cre-dependent viral vectors that express TVA, mapping of cell type-specific projection neurons can be achieved. However, there has not yet been a whole-brain tracing technology based on this system.

As a helper virus, the infectious properties of AAV depend on its capsid protein. AAV9 is widely used for neural circuit labelling because of its good effect on central nervous system infection among all AAV serotypes and has shown the ability to pass through the bloodbrain barrier (BBB) after peripheral vascular administration [12-14]. Deverman et al. [15] conceived a Cre recombination-based AAV directed evolution strategy to isolate a novel engineered AAV9 capsid, named PHP.B, with the insertion of a 7-amino-acid sequence TLAVPFK into the VP1 capsid protein, which was shown to outperform the standard AAV9 in transducing neurons after intravenous injection. Moreover, an enhanced version named PHP.eB is more efficient in crossing the bloodbrain barrier in C57BL/6 J mice and lowers the viral load required to transduce the majority of CNS neurons [16].

Through AAV vectors that can penetrate the bloodbrain barrier, whole-brain delivery of exogenous proteins can be achieved; thus, we designed AAV-PHP. eB-assisted retrograde tracing systems (PARTS) based on RV. Through the transduction of the TVA receptor using AAV-PHP.eB to the whole brain in C57BL/6 J mice, EnvA-pseudotyped defective rabies virus could label brain-wide upstream neurons of a defined brain area. Furthermore, combined with Cre transgenic mice, celltype-specific PARTS (cPARTS) was developed, which can retrograde label genetically defined brain-wide projection neurons.

The ventral tegmental area (VTA), a midbrain structure with multiple cell types, is known to integrate aversive and rewarding stimuli [17-21]. The VTA contains three types of neurons. The most abundant are dopaminergic neurons, which make up $60-65 \%$ of the total neurons, followed by GABAergic neurons, which make up approximately $30-35 \%$ of total neurons, and a small number of glutamatergic neurons, comprising approximately $2-3 \%$ of the total [22]. VTA GABA neurons have diverse functions, influencing dopaminergic activity through local inhibitory control and exerting dopamine (DA)independent effects through projections to distal brain regions [23]. As a proof of principle, we used PARTS and cPARTS to trace the upstream input of the VTA area. Our research provides new tools and technical support for the analysis of neural circuits.

\section{Results}

Establishment of a new retrograde tracing method

Receptor compensation can mediate more effective retrograde labelling of the virus. For example, canine adenovirus type-2 (CAV-2) has enhanced retrograde labelling efficiency by compensating the coxsackievirus and adenovirus receptor (CAR) [24]. After compensation of the AAV receptor (AAVR) in input neurons, AAV2 can transport the upstream connection that the previously reported AAV can hardly label [25]; TVA can mediate effective retrograde infection of EnvA envelope pseudotyped virus [26]. However, whether effective and broad-spectrum retrograde labelling can be achieved by whole-brain receptor compensation is unknown. To verify this strategy, we established a PARTS strategy, including a TVA-expressing AAV-PHP.eB, which can efficiently transduce brain neurons across the blood-brain barrier, and an EnvA envelope pseudotyped defective rabies virus that can specifically recognize and infect TVApositive neurons. Furthermore, we developed cell-typespecific PARTS (cPARTS), in which TVA expression is Cre-dependent.

Based on this design, Fig. 1 illustrates the functional elements carried in the viral vector in PARTS. We used AAV-PHP.eB carrying TVA fused with nuclear EGFP, and in cPARTS, TVA expression was Cre-dependent (Fig. 1A). The EnvA envelope pseudotyped SAD-B19$\triangle$ G-DsRed used in PARTS and cPRATS is shown in Fig. 1B.

\section{A new retrograde tracing method effectively labels upstream neurons}

AAV-PHP.eB can be used not only for whole brain infection by tail vein injection but also for in situ infection through local injection. Therefore, AAV-PHP.eB can also be used as a helper virus to analyse the connection between the two brain regions. To verify whether the helper virus can effectively mediate the retrograde labelling of EnvA-pseudotyped rabies virus, we tested PARTS (Fig. 2) and cPARTS (Fig. 3) in the $\mathrm{S} 1 \rightarrow \mathrm{CPu}$ circuit. rAAV-PHP.eB-hSyn::H2B-EGFP-T2A-TVA and 
A

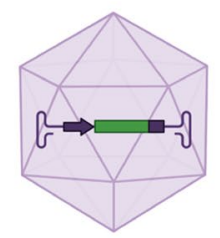

B

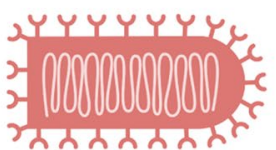

rAAV-PHP.eB-hSyn::H2B-EGFP-T2A-TVA-WPRE-hGH poly A

\begin{tabular}{llllllll} 
ITR hSyn Pro & $\mathrm{H} 2 \mathrm{~B}$ EGFP T2A TVA WPRE hGH PA ITR \\
\hline
\end{tabular}

rAAV-PHP.eB-hSyn::DIO-H2B-EGFP-T2A-TVA-WPRE-hGH poly A
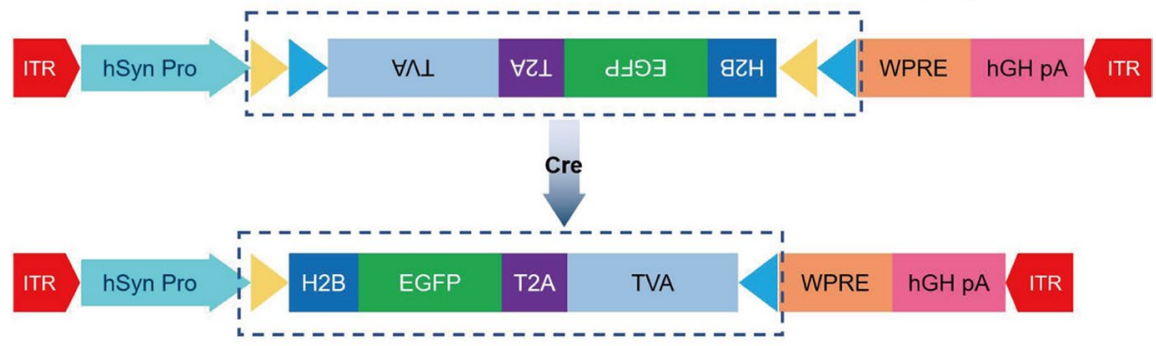

TR

Fig. 1 Schematic diagram of viral vectors carrying functional elements in PARTS and CPARTS strategies. A TVA-expressing vectors that can efficiently transduce brain neurons across the blood-brain barrier. The expression is direct and Cre-dependent. The AAV serotype used here is PHP.eB. B EnvA-pseudotyped glycoprotein (G)-defective rabies virus that can specifically recognize and infect TVA-positive neurons

rAAV-PHP.eB-hSyn::DIO-H2B-EGFP-T2A-TVA were injected into the $\mathrm{S} 1$ of $\mathrm{C} 57 \mathrm{BL} / 6 \mathrm{~J}$ mice and Thy1-Cre transgenic mice, respectively, and RV-EnvA-SAD-B19$\Delta \mathrm{G}$-DsRed was injected into the $\mathrm{CPu}$ after 2 weeks. The mice were sacrificed after another week, and the cortical region projecting to the $\mathrm{CPu}$ was imaged by an Olympus VS120 Slide Scanner microscope (Figs. 2A, 3A). Both green and red fluorescent signals appeared in S1, and red fluorescence was colabelled with green fluorescence (Figs. 2B, 3B). Moreover, no DsRed fluorescent signals of RV-EnvA were observed in the injection site $\mathrm{CPu}$. These results showed that PARTS and cPARTS could retrogradely label projection neurons after injection into specific brain areas.

\section{Use of the new retrograde tracing method to trace the broad-spectrum upstream input network of the VTA}

To verify whether PARTS can be used for brain-wide retrograde tracing, rAAV-PHP.eB-hSyn-H2B-EGFP-T2ATVA was intravenously injected into C57BL/6 J adult mice. At 3 weeks postinjection, RV-EnvA-SAD-B19- $\Delta$ GDsRed was injected into the VTA, the mice were sacrificed after another week, and the upstream inputs of the VTA were imaged (Fig. 4A). We found that RV-EnvASAD-B19- $\Delta$ G-DsRed could infect the injection site
(Fig. 4B) and upstream brain regions (Fig. 4C), including the cerebral cortex (CTX), lateral habenula ( $\mathrm{LHb})$, lateral hypothalamic area (LHA), zona incerta (ZI), midbrain reticular nucleus (MRN), and cerebellar nuclei (CBN), among others. More than $96 \%$ of rabies-labelled cells are also positive for AAV-TVA tags (EGFP), and several cells have only red signals because the weak expression of the TVA receptor can result in RV-EnvA infection; in this case, EGFP signals are not significant [10]. These results indicate that PARTS can be used to retrograde trace brain-wide input neurons of the specific brain regions of our choice.

\section{Use of the new retrograde tracing method to label} the cell-type-specific input network upstream of the VTA Before using the cPARTS method to trace the cell-typespecific upstream input network of the VTA, rAAVPHP.eB-hSyn-DIO-H2B-EGFP-T2A-TVA, which has Cre-dependent conditional EGFP expression, was intravenously injected into VGAT-Cre mice, and almost no green fluorescence signals of GABAergic neurons in the thalamus were labelled (Figs. 5C, D), consistent with the in situ hybridization (ISH) results published on the Allen Brain Atlas website. As a control, rAAV-PHP.eB-hSynH2B-EGFP-T2A-TVA was intravenously injected into 


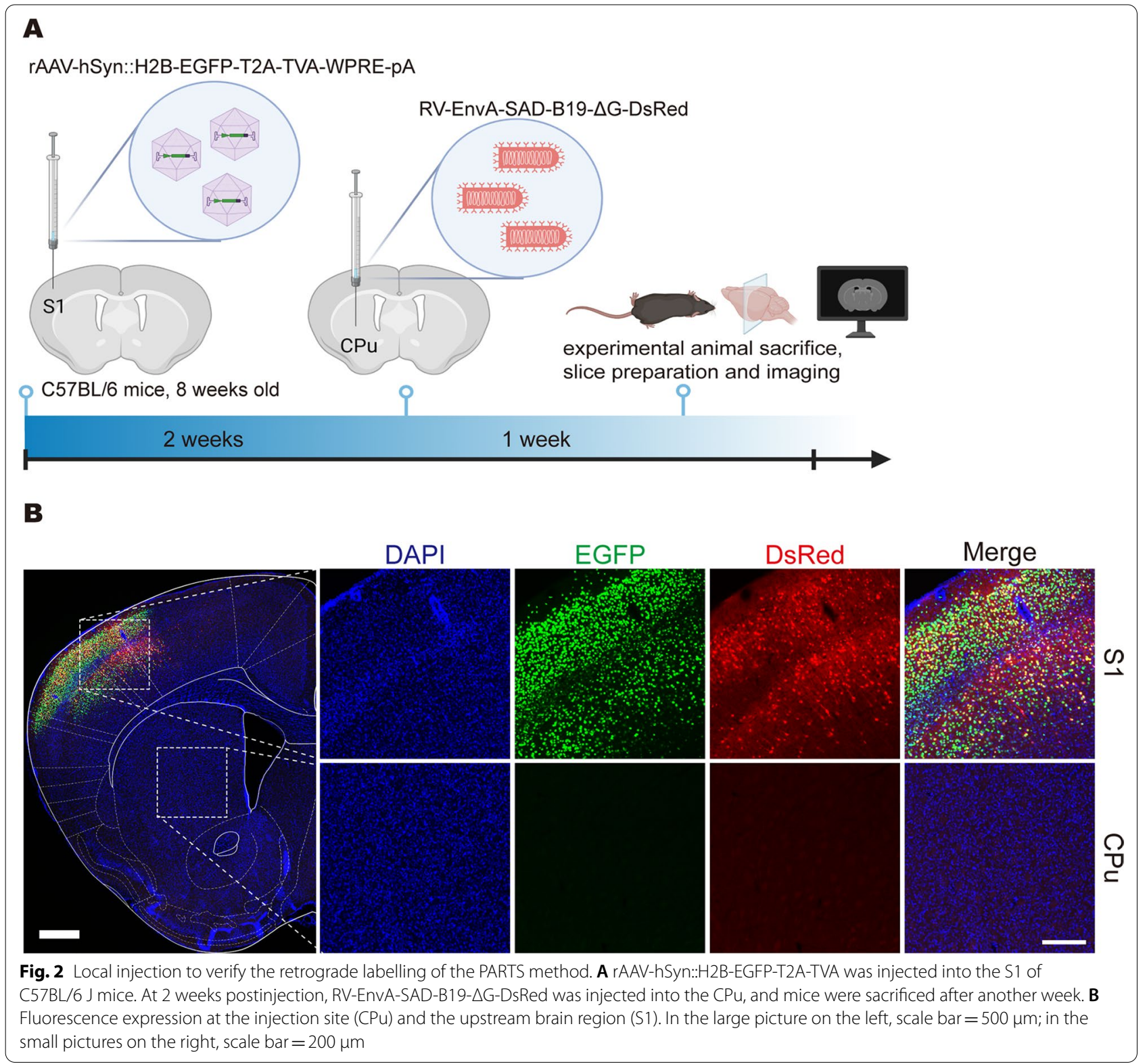

C57BL/6 J mice, and obvious green fluorescence signals could be observed in the thalamus of $\mathrm{C} 57 \mathrm{BL} / 6 \mathrm{~J}$ mice (Fig. 5A, B). These results showed that rAAV-PHP.eBhSyn-DIO-H2B-EGFP-T2A-TVA could specifically label GABAergic neurons in the whole brains of VGAT-Cre mice. After rAAV-PHP.eB-hSyn-H2B-EGFP-T2A-TVA was intravenously injected into adult $\mathrm{C} 57 \mathrm{BL} / 6 \mathrm{~J}$ mice for 3 weeks, RV-EnvA-SAD-B19- $\Delta$ G-DsRed was injected into the VTA, the mice were sacrificed after another week, and the upstream inputs of the VTA were imaged (Fig. 6A). We found that RV-EnvA-SAD-B19- $\Delta$ G-DsRed could infect the injection site (Fig. 6B) and upstream brain regions (Fig. 6C), including the globus pallidus, external segment (GPe), lateral hypothalamic area (LHA), zona incerta $(\mathrm{ZI})$, pontine reticular nucleus (PRNr), laterodorsal tegmental nucleus (LDTg) and vestibular nuclei (VNC), among others. Moreover, fewer than five cells in a single brain area showed single-positive neurons of DsRed, which may be caused by low levels of TVA expression. These levels of leak might be undetectable by conventional methods such as fluorescent protein expression but can result in RV infection and high levels of viral gene expression [10, 27-29]. These results indicate that cPARTS can be used to retrograde trace brainwide genetically defined input neurons of the specific brain regions of our choice. 


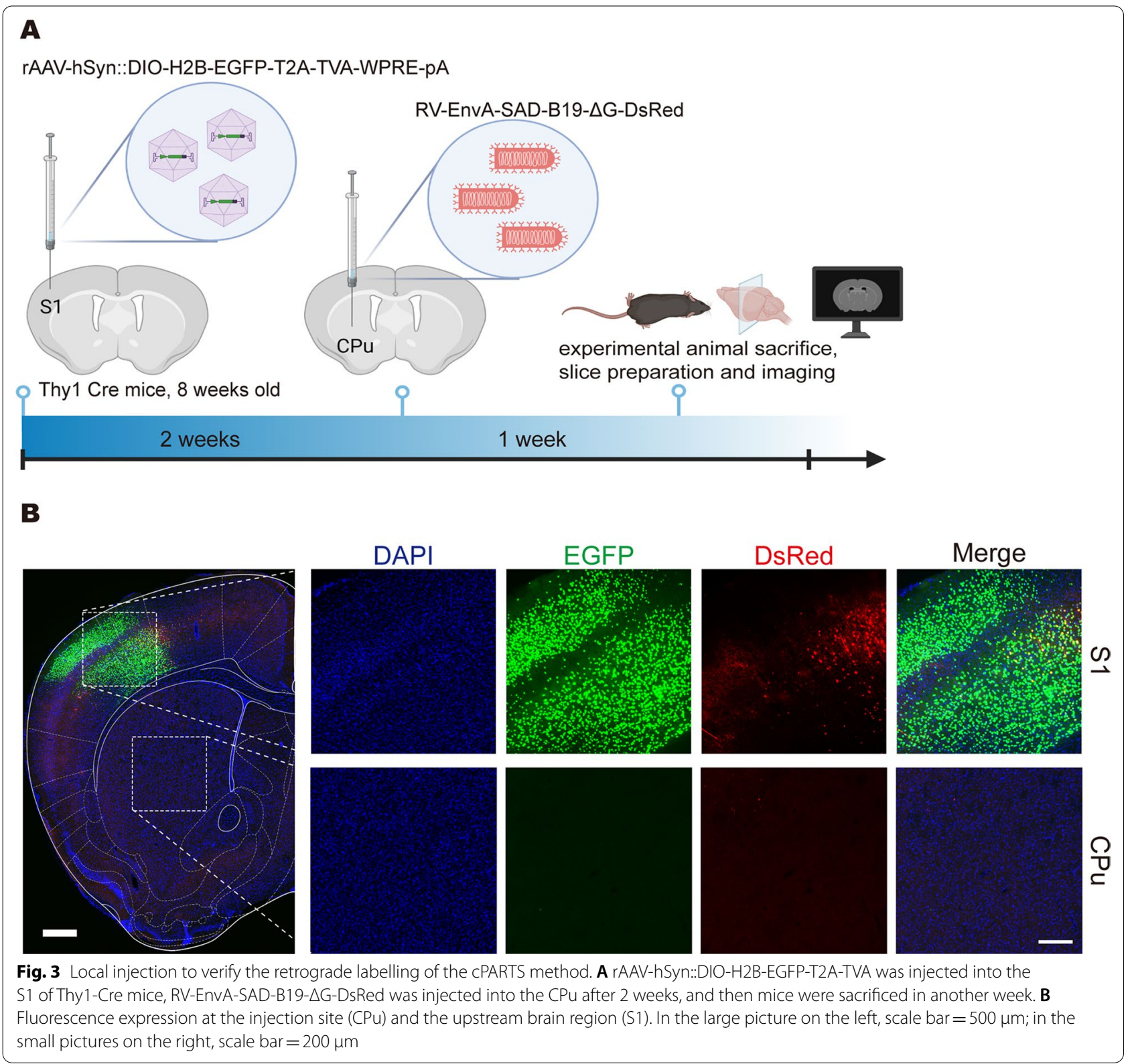

\section{New strategies for retrograde tracing}

Based on the above experimental results, we successfully established new retrograde tracing technologies, including PARTS and cPARTS. Their application strategies are summarized as follows: PARTS identifies neurons in the $\mathrm{B}$ region (local injection, Fig. 7A) or in brain-wide regions (intravenous injection, Fig. 7C) that project to a specific A region; cPARTS identifies celltype-specific neurons in the $\mathrm{B}$ region (local injection, Fig. 7B) or in brain-wide regions (intravenous injection, Fig. 7D) that project to a specific A region.

\section{(See figure on next page.)}

Fig. 4 Use of the PARTS method to trace the broad-spectrum upstream input network of the VTA. A Schematic diagram of retrograde tracing by the PARTS method. Helper virus that expresses the TVA receptor fused with nuclear EGFP was intravenously injected into C57BL/6 J adult mice, and RV-EnvA-SAD-B19- $\triangle$ G-DsRed was injected into the VTA after 3 weeks. Then, the mice were sacrificed after another week, and the upstream inputs of the VTA were imaged. $\mathbf{B}$ Fluorescence expression at the injection site (VTA), scale bar $=200 \mu \mathrm{m}$. C Fluorescence expression in some upstream brain regions of the VTA. Red fluorescence signals were colabelled with green fluorescence signals, scale bar $=200 \mu \mathrm{m}$. CTX cerebral cortex, $L H b$ lateral habenula, LHA lateral hypothalamic area, ZI zona incerta, MRN midbrain reticular nucleus, CBN cerebellar nuclei 
$\mathbf{A}$

rAAV-PHP.eB-hSyn::H2B-EGFP-T2A-TVA-WPRE-pA

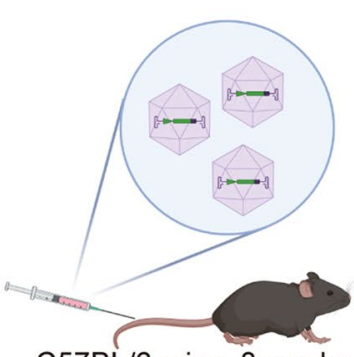

C57BL/6 mice, 8 weeks old

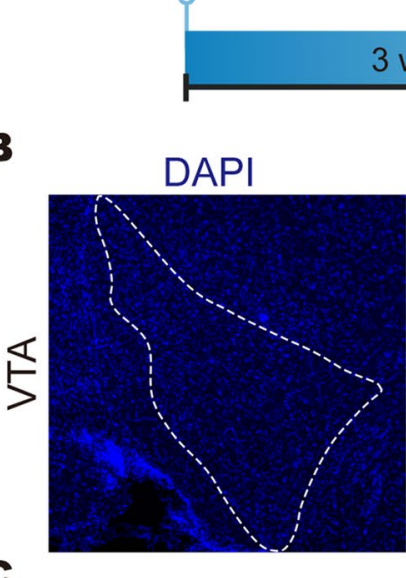

3 weeks

RV-EnvA-SAD-B19- $\triangle$ G-DsRed

B

C

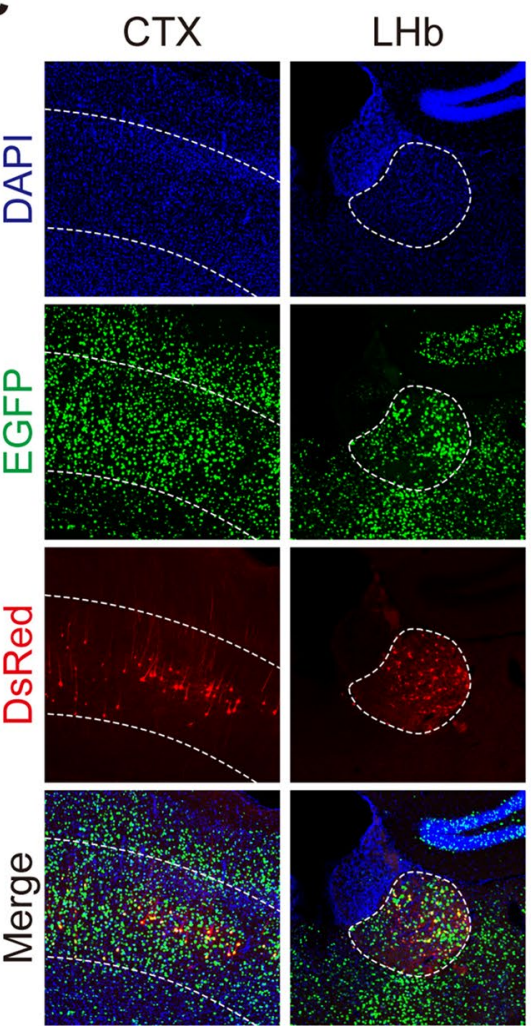

EGFP
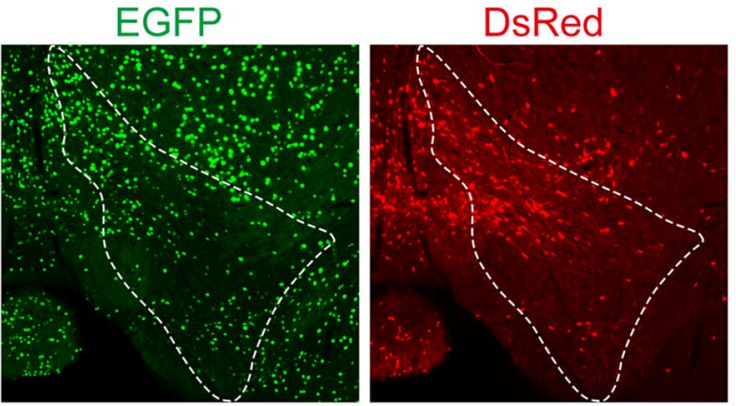

experimental animal sacrifice, slice preparation and imaging

1 week i
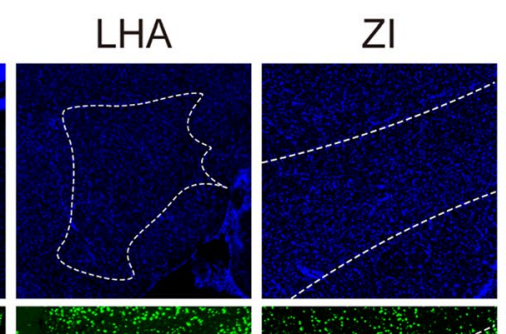

MRN

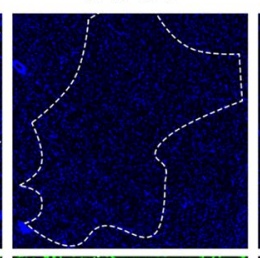

CBN
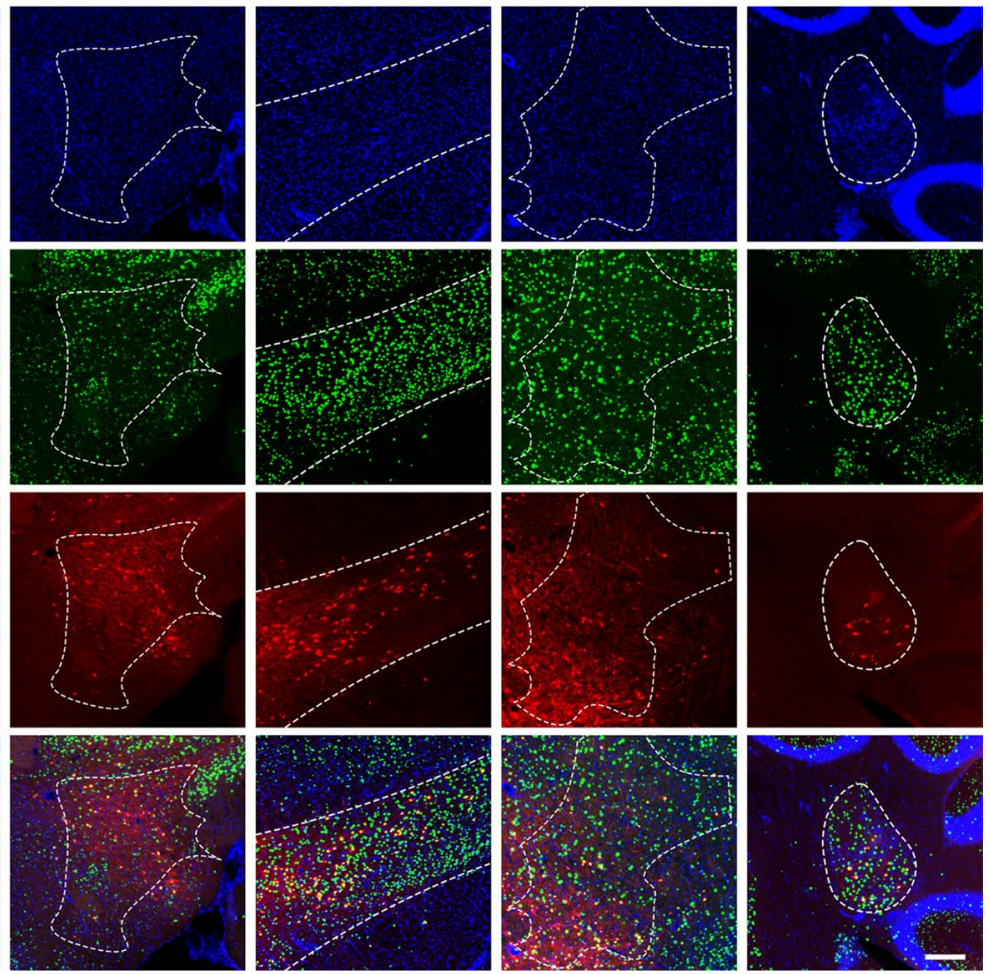

Fig. 4 (See legend on previous page.) 

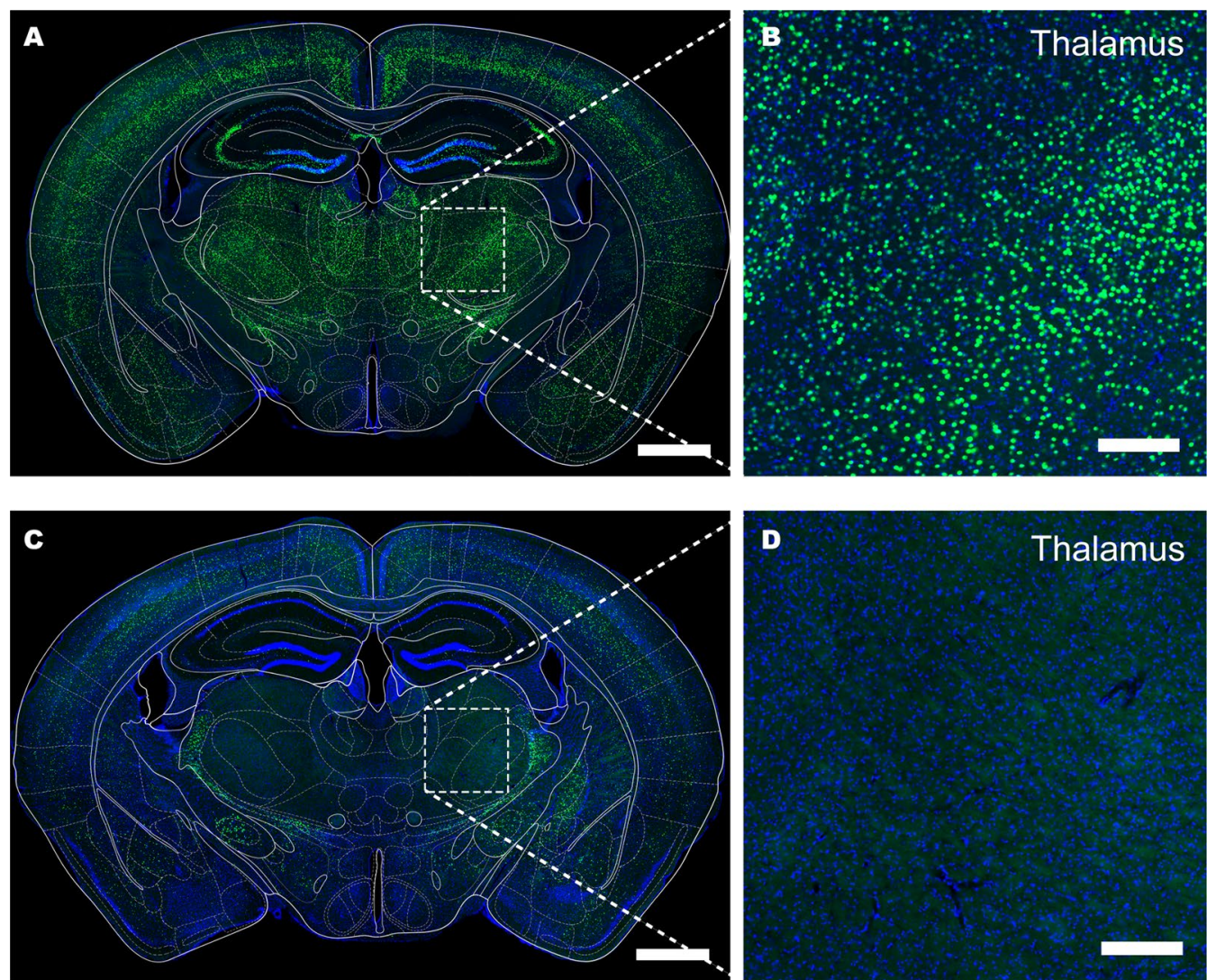

Fig. 5 Distribution of GABAergic neurons in the whole brain. A Labelling of rAAV-PHP.eB-hSyn-H2B-EGFP-T2A-TVA in C57BL/6 J mice, scale bar $=1 \mathrm{~mm}$. B Green fluorescence signals in the thalamus of C57BL/6 J mice, scale bar $=200 \mu \mathrm{m}$. C Conditional labelling of rAAV-PHP. eB-hSyn-DIO-H2B-EGFP-T2A-TVA in VGAT-Cre transgenic mice, scale bar $=1 \mathrm{~mm}$. (D) Green fluorescence signals in the thalamus of VGAT-Cre mice, scale $b a r=200 \mu \mathrm{m}$

\section{Discussion}

Retrograde neurotropic viruses, which permit efficient gene transfer to the cell bodies of projection neurons from axonal terminals, have become essential tracers in the field of neuroscience by enabling the structural and functional analysis of genetically defined neural circuits. Due to their use of different receptors and infection mechanisms, the labelling characteristics of the various viruses are quite different [30,31]. Such tropism is useful for the study of specific neural circuits, but in general, it lacks universality $[3,24]$. In the present study, we developed PARTS and cPARTS strategies for the analysis of brain-wide projection neurons received by specific brain regions. AAV-PHP.eB, which can efficiently cross the blood-brain barrier in C57BL/6 J mice [16], is applied to the RV retrograde tracing system through whole-brain TVA compensation after intravenous injection, and broad-spectrum or cell-type-specific retrograde labelling in the whole brain can be achieved by EnvA-pseudotyped glycoprotein-deleted rabies virus. The retrograde tracing technique in two brain regions (Fig. 7A, B) can be widely used in the analysis of single neural circuits; moreover, our new methods can expand the labelling system to the brain-wide scale (Fig. 7C, D).

\section{(See figure on next page.)}

Fig. 6 Use of the CPARTS method to trace the cell-type-specific upstream input network of the VTA. A Schematic diagram of retrograde tracing by the CPARTS method. Cre-dependent helper virus that expresses the TVA receptor fused with nuclear EGFP was intravenously injected into VGAT-Cre adult mice, and RV-EnvA-SAD-B19- $\triangle$ G-DsRed was injected into the VTA after 3 weeks. Then, the mice were sacrificed after another week, and the upstream inputs of the VTA were imaged. B Fluorescence expression at the injection site (VTA), scale bar $=200 \mu \mathrm{m}$. C Fluorescence expression in some upstream brain regions of the VTA. Red fluorescence signals were colabelled with green fluorescence signals, scale bar $=200 \mu \mathrm{m}$. GPe globus pallidus, external segment; LHA lateral hypothalamic area; ZI zona incerta; PRNr pontine reticular nucleus; LDTg laterodorsal tegmental nucleus; VNC vestibular nuclei 
A

rAAV-PHP.eB-hSyn::DIO-H2B-EGFP-T2A-TVA-WPRE-hGH poly A

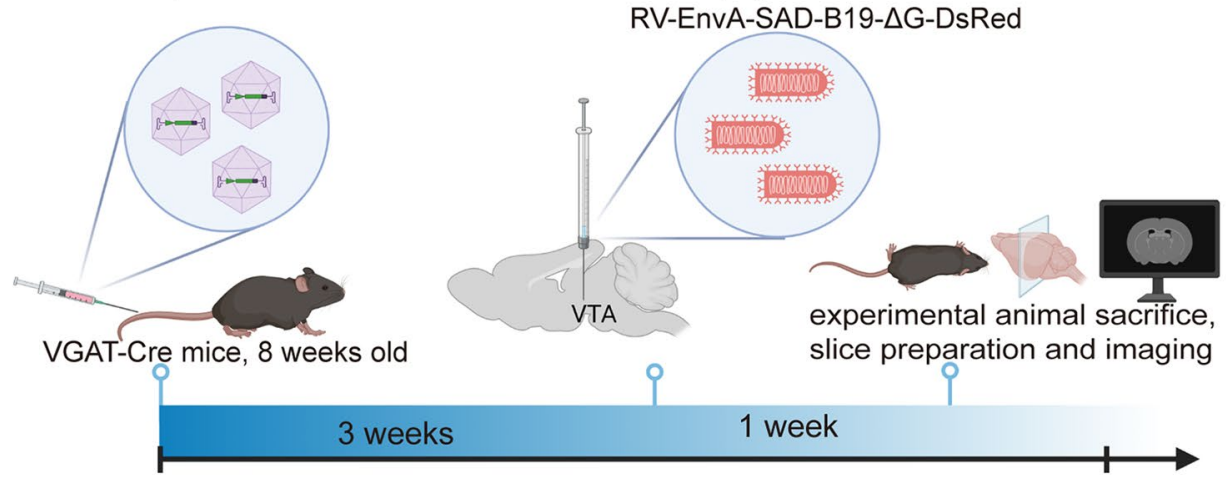

B
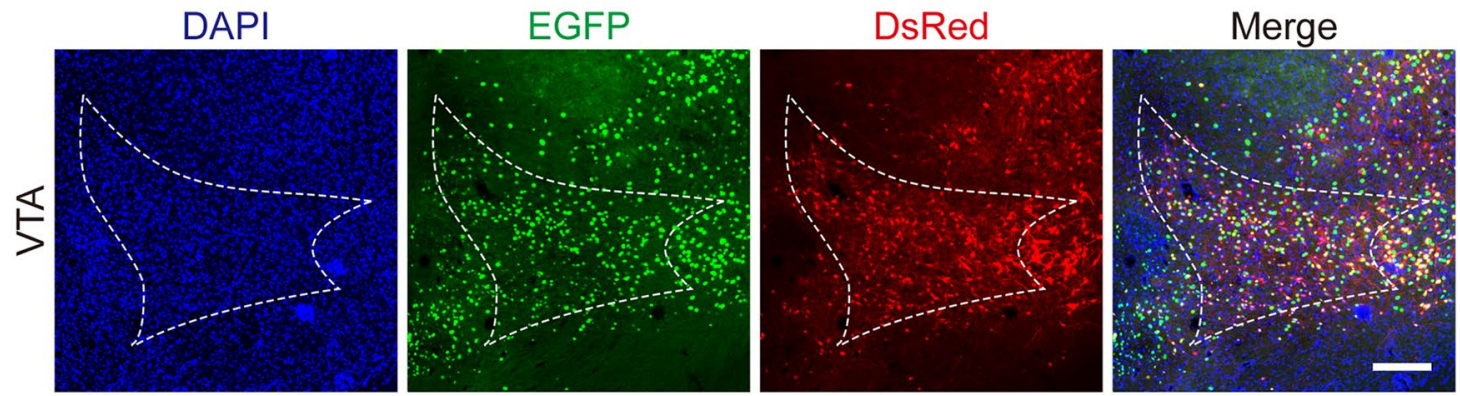

C

$\mathrm{GPe}$

LHA

ZI

$\mathrm{PRNr}$

LDTg

VNC
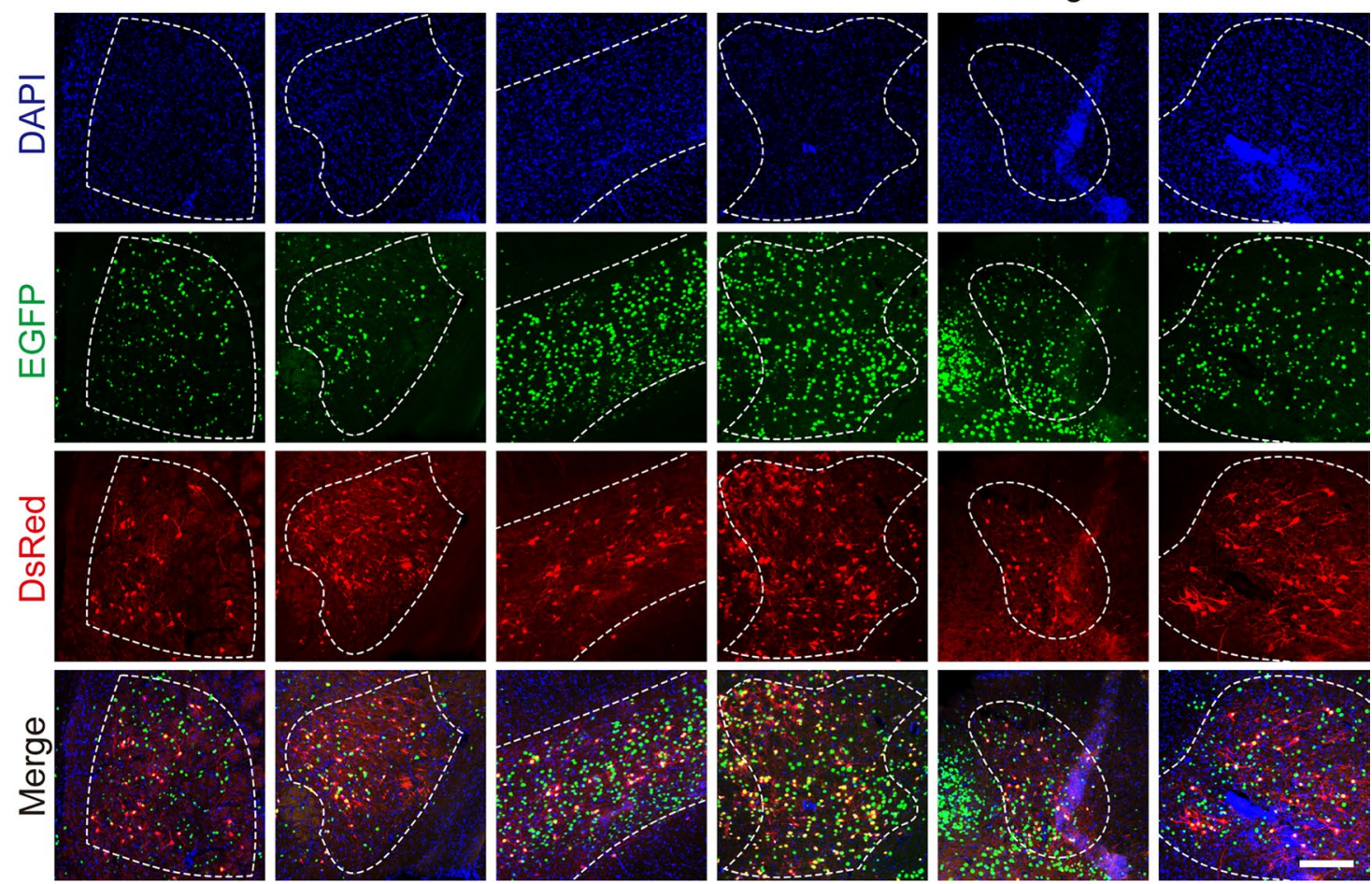

Fig. 6 (See legend on previous page.) 


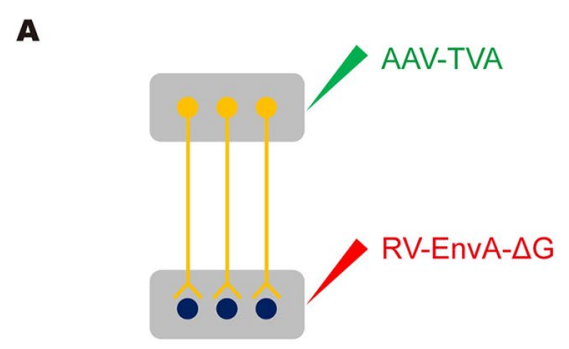

$\mathbf{B}$
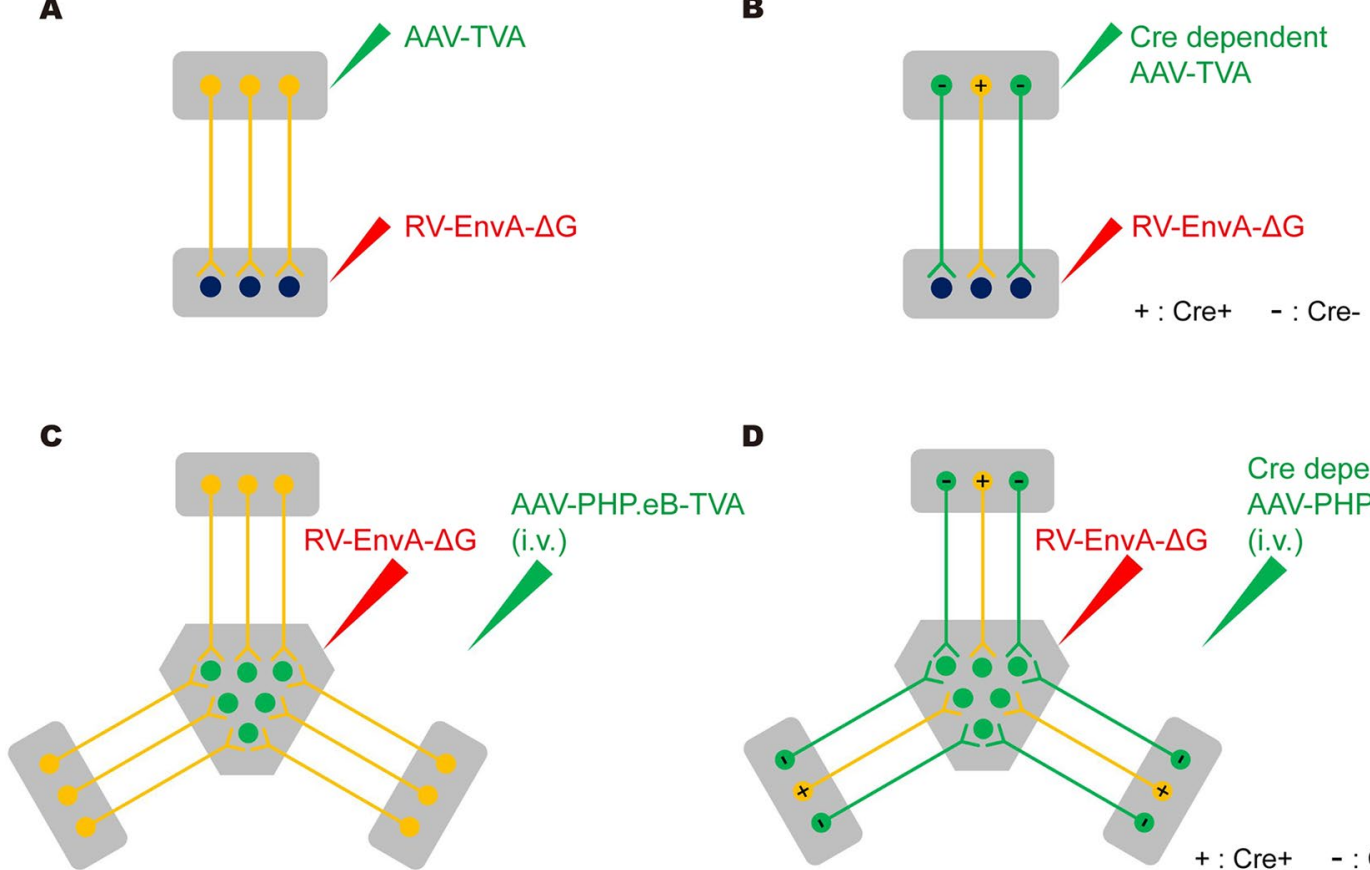

D

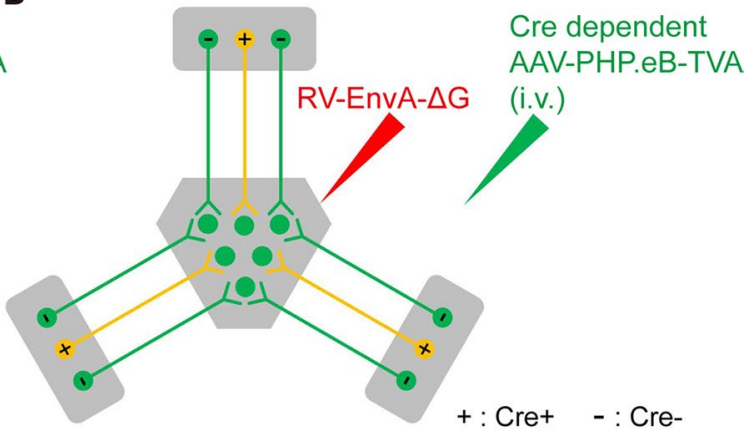

Fig. 7 Summary of retrograde tracing strategies. A The PARTS strategy can realize traditional broad-spectrum retrograde tracing in two brain regions. B The CPARTS strategy can realize traditional cell type-specific retrograde tracing in two brain regions. C The PARTS strategy can realize broad-spectrum retrograde tracing in the whole brain. D The CPARTS strategy can realize cell type-specific retrograde tracing in the whole brain

Some natural and engineered retrograde neurotropic viruses have been widely used in the study of neural circuits, such as rabies virus (RABV) [3, 7], lentivirus (LV) packaged by modified RVG [32-35], canine adenovirus (CAV) [36], herpes simplex virus (HSV) [37], and adenoassociated virus (AAV) $[1,38-40]$. However, they have different labelling efficiencies and brain region selectivities $[3,26]$. Modifying the surface structures of the viral envelope or capsid can improve tropism, but viruses still rely on cell surface molecules for uptake and transport and cannot gain entry into cells without the required receptors [24]. Receptor complementation approaches are a better strategy to overcome tropism and mediate more effective retrograde labelling of the virus. For example, canine adenovirus type-2 (CAV-2) has enhanced retrograde labelling efficiency by compensating for the coxsackievirus and adenovirus receptor (CAR) [24]. After compensation of the AAV receptor (AAVR) in input neurons, AAV2 can transport the upstream connection that the previously reported AAV can hardly label [25]; TVA can mediate effective retrograde infection of EnvA envelope pseudotyped virus [26]. Among them, TVA compensation for EnvA-pseudotyped virus permits retrograde labelling of cell-type-specific inputs because viruses pseudotyped with the avian sarcoma leucosis virus glycoprotein EnvA can infect only neurons that express exogenous TVA receptor, an avian receptor protein that is absent in mammalian cells [9]. Therefore, TVA receptor compensation for neurotropic viruses may be valuable tracers for targeting and manipulating broadspectrum or genetically defined neural input networks.

We verified the whole-brain compensation effect of TVA carried by PHP.eB in C57BL/6 J and VGAT-Cre transgenic mice, and the distribution of GABAergic neurons in the whole brain was consistent with the in situ hybridization (ISH) results published on the Allen Brain Atlas website. We verified the PARTS and cPARTS labelling effects of brain-wide retrograde tracing in the VTA region, and significant fluorescence signals could be seen in several upstream regions (Figs. 4C, 6C). Among them, LHb is involved in reward, aversion, addiction and depression through descending interactions with several brain structures, including the ventral tegmental area (VTA) [41]. The LHA is implicated in feeding, food reward, and other motivated behaviours, and activation of all VTA-projecting LHA neurons enhances sucrose seeking [42]. de Git et al. [43] confirmed that activity of the ZI to VTA projection promotes feeding, which improves the understanding of the neurobiology of feeding behaviour and body weight regulation. Hernandez et al. [44] proved that GLP-1 receptor signalling in the LDTg attenuates cocaine seeking by activating 
GABAergic circuits that project to the VTA. Therefore, PARTS and cPARTS can be applied to analyse projection neurons related to important behaviours.

The VTA, which is an important brain region and is involved in various behaviours, is reported to connect with most brain regions; however, the upstream inputs of distinct cell types have not yet been completely defined. Based on the PARTS and cPARTS strategies, we can provide more information and anatomical evidence to help understand the precise connections and diverse functions of specific brain regions. Among the commonly used retrograde tracing tools, AAV2-retro has difficulty achieving unbiased retrograde labelling and prefers to infect cortical neurons; N2cG-RV- $\Delta \mathrm{G}$ can only broad-spectrum retrograde infect projection networks that are not cell type-specific [3]. The PARTS/cPARTS strategies overcome the shortcomings of the above two. On the basis of broad-spectrum labelling, cell type-specific retrograde tracing can be easily achieved using transgenic animals and conditionally expressed TVA elements carried by AAV-PHP.eB. In addition, many different viral tracers or methods for retrograde tracing have been used in the neuroscience field. Here, we provide a more detailed comparison (pros and cons) among them (Table 1), and researchers can choose the appropriate viral tracers according to their different experimental needs.

Whole-brain delivery of the TVA receptor depends on the AAV mutant PHP.eB, which efficiently crosses the blood-brain barrier, but the neurotropic properties of AAV-PHP.eB are limited to C57BL/6 J mice [45, 46]; therefore, expanding the scope of application of PARTS currently requires the acquisition of AAV mutants that efficiently cross the blood-brain barrier in a broader range of species. According to previous reports, intravenous administration of $1 \times 10^{11} \mathrm{vg}$ of AAV-PHP.eB transduced $69 \%$ of cortical and $55 \%$ of striatal neurons in adult

Table 1 Comparison of viral tracers and methods of retrograde tracing

\begin{tabular}{llllll}
\hline Method/virus & Efficiency & Preparation & Toxicity & $\begin{array}{l}\text { Brain region } \\
\text { selectivity }\end{array}$ & $\begin{array}{l}\text { Cell- } \\
\text { type- } \\
\text { specific }\end{array}$ \\
\hline AAV2-retro & High & Easy & Low & Yes & Yes \\
AAV \&AAVR & Medium & Easy & Low & No & Yes \\
CAV2 & Low & Easy & Low & Yes & Yes \\
CAV2 \& CAR & Medium & Easy & Low & No & Yes \\
RV- $\triangle G(N 2 C G)$ & High & Easy & High & No & No \\
PARTS & High & Easy & High & No & No \\
CPARTS & High & Easy & High & No & Yes \\
\hline
\end{tabular}

Efficiency and brain region selectivity of viral tracers are relative to other viruses, and in most cases, researchers can choose the appropriate viral tracers according to their different experimental needs mice [16]. Although the dosage was increased, intravenous delivery of AAV-PHP.eB could only transduce a fraction of whole neuron populations. This limitation may be compensated by the development of AAV with higher infection efficiency.

In summary, we have proven that brain-wide TVA compensation can mediate efficient retrograde targeting of the RV to projection neurons. Our research provides new tools and technical support for the analysis of neural circuits.

\section{Materials and methods}

Vector construction and virus preparation

To construct pAAV-PHP.eB-hSyn-DIO-H2B-EGFP-T2ATVA-WPRE-pA, the H2B-EGFP-T2A-TVA sequence was synthesized and inserted into pAAV-hSyn-DIOEGFP-WPRE-hGH polyA (BrainCase, ShenZhen) digested by the restriction enzymes NheI and AscI (New England Biolabs). To construct the pAAV-PHP. eB-hSyn-H2B-EGFP-T2A-TVA-WPRE-pA, the H2BEGFP-T2A-TVA sequence was amplified and inserted into the pAAV-hSyn-DIO-EGFP-WPRE-hGH polyA digested by the restriction enzymes Sall and HindIII (New England Biolabs). AAV vectors, including rAAVPHP.eB-hSyn-DIO-H2B-EGFP-T2A-TVA-WPRE-pA and rAAV-PHP.eB-hSyn-H2B-EGFP-T2A-TVA-WPRE-pA, were produced in HEK-293 $\mathrm{T}$ cells cotransfected with pUCmini-iCAP-PHP.eB (Addgene plasmid \#103005) and pAdDeltaF6 (Addgene plasmid \#112867) and then purified by iodixanol gradient ultracentrifugation [47]. The purified rAAVs were titered by qPCR using the iQ SYBR Green Supermix kit (Bio-Rad). The titers of rAAV-PHP. eB-hSyn-H2B-EGFP-T2A-TVA-WPRE-pA and rAAVPHP.eB-hSyn-DIO-H2B-EGFP-T2A-TVA-WPRE-pA were $5.28 \times 10^{12} \mathrm{VG} / \mathrm{mL}$ and $1.1 \times 10^{13} \mathrm{VG} / \mathrm{mL}$, respectively. RV-EnvA-SAD-B19- $\Delta$ G-DsRed was prepared according to a previously reported method [48], and the titer was $5 \times 10^{7} \mathrm{IU} / \mathrm{mL}$.

\section{Animals}

Adult male (8-10 weeks old) C57BL/6 J mice (Hunan SJA Laboratory Animal Company) and VGAT-Cre transgenic mice (The Jackson Laboratory) were used for all experiments. The mice were housed in the appropriate environment with a 12/12-h light/dark cycle, and water and food were supplied ad libitum. All surgical and experimental procedures were performed in accordance with the guidelines formulated by the Animal Care and Use Committee of the Innovation Academy for Precision Measurement Science and Technology, Chinese Academy of Sciences. 


\section{Virus injection}

The stereotactic injection coordinates were selected according to Paxinos and Franklin's The Mouse Brain in Stereotaxic Coordinates, 4th edition [49]. The stereotactic coordinates for S1 were as follows: AP: $+0.50 \mathrm{~mm}$; $\mathrm{ML}: \pm 3.00 \mathrm{~mm}$; DV: $-2.00 \mathrm{~mm}$ from bregma. The $\mathrm{CPu}$ values were as follows: AP: $+0.75 \mathrm{~mm}$; $\mathrm{ML}: \pm 2.00 \mathrm{~mm}$; and DV: - $3.30 \mathrm{~mm}$ from bregma. The VTA values were as follows: AP: $-3.20 \mathrm{~mm}$; ML: $\pm 0.45 \mathrm{~mm}$; DV: $-4.30 \mathrm{~mm}$ from bregma. Eight- to ten-weekold C57BL/6 J mice and VGAT-Cre transgenic mice (20-25 g) were used for virus injection, and the standard injection process was performed as previously reported [3]. After 2 weeks of AAV virus expression in S1, RV-EnvA-SAD-B19- $\Delta$ G-DsRed was injected into the $\mathrm{CPu}$ of $\mathrm{C} 57 \mathrm{BL} / 6 \mathrm{~J}$ mice, and the mice were sacrificed at 7 days postinjection using the conventional cardiac perfusion method. For brain-wide retrograde tracing, TVA-expressing rAAV-PHP.eB-hSyn-DIO-H2B-EGFPT2A-TVA and rAAV-PHP.eB-hSyn-H2B-EGFP-T2ATVA $\left(4 \times 10^{11} \mathrm{VG} /\right.$ mouse $)$ were intravenously injected into VGAT-Cre transgenic adult mice and C57BL/6 J mice, respectively. After 3 weeks, RV-EnvA-SAD-B19$\Delta$ G-DsRed was injected into the VTA, and the mice were sacrificed at 7 days postinjection.

\section{Slice preparation and imaging}

Slice preparation and imaging were completed according to previously reported methods [4]. The brains were soaked with $4 \%$ paraformaldehyde solution overnight, dehydration was accomplished at $37{ }^{\circ} \mathrm{C}$ with $30 \%$ sucrose solution, the brain was sectioned at a thickness of $40 \mu \mathrm{m}$ using a frozen section instrument, and the brain slices were retrieved at $200-\mu \mathrm{m}$ intervals. The brain slices were washed 3 times with phosphate-buffered saline (PBS) for 10 min each time. After 4,6-diamidino-2-phenylindole (DAPI) staining for $10 \mathrm{~min}$, the brain slices were applied neatly on microscope slides and washed with PBS 3 times, followed by sealing with $70 \%$ glycerol. Imaging was performed using an Olympus VS120 Slide Scanner microscope (Olympus, Japan).

\section{Abbreviations}

AAV: Adeno-associated virus; PARTS: AAV-PHP.eB assisted retrograde tracing systems; CPARTS: Cell-type-specific PARTS; VG: Viral genomes; IU: Infection units, DAPI: 4,6-diamidino-2-phenylindole; CPu: Caudate putamen (striatum); CTX: Cerebral cortex; LHb: Lateral habenula; LHA: Lateral hypothalamic area; ZI: Zona incerta; MRN: Midbrain reticular nucleus; CBN: Cerebellar nuclei; VTA: Ventral tegmental area; GPe: Globus pallidus, external segment; PRNr: Pontine reticular nucleus; LDTg: Laterodorsal tegmental nucleus; VNC: Vestibular nuclei; $\mathrm{TH}$ :Thalamus.
}

Academy of Sciences) for technical assistance with centrifugation and microscope imaging. Schematic diagrams of the process in the figures of this article were created with BioRender.com.

\section{Authors' contributions}

$\mathrm{KL}$ and FX contributed to the study idea and design; FX, CY and JW contributed to funding acquisition and resources; ZH, NL, JK, LL, ZX and SW performed the experiments and data acquisition; $\mathrm{ZH}, \mathrm{NL}, \mathrm{KL}$ and $\mathrm{YW}$ performed the data analysis; $\mathrm{ZH}, \mathrm{KL}$ and $\mathrm{FX}$ drafted the manuscript and contributed to its review and editing. All authors read and approved the final manuscript.

\section{Funding}

This work was supported by the Key-Area Research and Development Program of Guangdong Province (2018B030331001), the Strategic Priority Research Program of the Chinese Academy of Sciences (XDB32030200), the National Natural Science Foundation of China (31830035, 31771156, 21921004), the Shenzhen Key Laboratory of Viral Vectors for Biomedicine (ZDSYS20200811142401005), and the National Key R\&D Program of China (No. 2018YFC1704600).

\section{Availability of data and materials}

The datasets used or analysed during the current study are available from the corresponding author on reasonable request.

\section{Declarations}

\section{Ethics approval and consent to participate}

All procedures used were approved by the Animal Care and Use Committees at the Innovation Academy for Precision Measurement Science and Technology, Chinese Academy of Sciences. All experiments with viruses were performed in a BSL2/ABSL2 laboratory and animal facilities.

\section{Consent for publication}

Not applicable.

\section{Competing interests}

The authors declare that there are no conflicts of interest between them.

\section{Author details}

${ }^{1}$ Key Laboratory of Magnetic Resonance in Biological Systems, State Key Laboratory of Magnetic Resonance and Atomic and Molecular Physics, National Center for Magnetic Resonance in Wuhan, Wuhan Institute of Physics and Mathematics, Innovation Academy for Precision Measurement Science and Technology, Chinese Academy of Sciences-Wuhan National Laboratory for Optoelectronics, Wuhan 430071, People's Republic of China. ${ }^{2}$ University of Chinese Academy of Sciences, Beijing 100049, People's Republic of China. ${ }^{3}$ The Brain Cognition and Brain Disease Institute (BCBDI), Shenzhen Key Laboratory of Viral Vectors for Biomedicine, Shenzhen Institute of Advanced Technology, Chinese Academy of Sciences, Shenzhen 518055, People's Republic of China. ${ }^{4}$ Shenzhen-Hong Kong Institute of Brain Science-Shenzhen Fundamental Research Institutions, NMPA Key Laboratory for Research and Evaluation of Viral Vector Technology in Cell and Gene Therapy Medicinal Products, Shenzhen, Key Laboratory of Quality Control Technology for VirusBased Therapeutics, Guangdong Provincial Medical Products Administration, Shenzhen 518055, People's Republic of China. ${ }^{5}$ Wuhan National Laboratory for Optoelectronics, Huazhong University of Science and Technology, Wuhan 430074, People's Republic of China. ${ }^{6}$ Department of Pathophysiology, Key Lab of Neurological Disorder of Education Ministry, School of Basic Medicine, Tongji Medical College, Huazhong University of Science and Technology, Wuhan, People's Republic of China. ${ }^{7}$ College of Life Sciences, Wuhan University, Wuhan, People's Republic of China. ${ }^{8}$ HongYi Honor College, Wuhan University, Wuhan, People's Republic of China. ${ }^{9}$ Shenzhen-Hong Kong Institute of Brain Science-Shenzhen Fundamental Research Institutions, Shenzhen 518055, People's Republic of China. ${ }^{10}$ Center for Excellence in Brain Science and Intelligence Technology, Chinese Academy of Sciences, Shanghai 200031, People's Republic of China.

\section{Acknowledgements}

We are grateful to Liting Luo and Lingling Xu (Core Facility Center, Innovation Academy for Precision Measurement Science and Technology, Chinese 
Received: 26 October 2021 Accepted: 16 January 2022

Published online: 29 January 2022

\section{References}

1. Tervo DG, Hwang BY, Viswanathan S, Gaj T, Lavzin M, Ritola KD, et al. A designer AAV variant permits efficient retrograde access to projection neurons. Neuron. 2016:92(2):372-82.

2. Wouterlood FG, Bloem B, Mansvelder HD, Luchicchi A, Deisseroth K. A fourth generation of neuroanatomical tracing techniques: exploiting the offspring of genetic engineering. J Neurosci Methods. 2014;235:331-48.

3. Zhu X, Lin K, Liu Q, Yue X, Mi H, Huang X, et al. Rabies virus pseudotyped with CVS-N2C glycoprotein as a powerful tool for retrograde neuronal network tracing. Neurosci Bull. 2020;36(3):202-16.

4. Lin $\mathrm{K}$, Zhong $X$, Li L, Ying M, Yang T, Zhang Z, et al. AAV9-Retro mediates efficient transduction with axon terminal absorption and blood-brain barrier transportation. Mol Brain. 2020;13(1):138.

5. Vercelli A, Repici M, Garbossa D, Grimaldi A. Recent techniques for tracing pathways in the central nervous system of developing and adult mammals. Brain Res Bull. 2000;51(1):11-28.

6. Katz LC. Local circuitry of identified projection neurons in cat visual cortex brain slices. J Neurosci. 1987;7(4):1223-49.

7. Wickersham IR, Finke S, Conzelmann KK, Callaway EM. Retrograde neuronal tracing with a deletion-mutant rabies virus. Nat Methods. 2007:4(1):47-9.

8. Suzuki L, Coulon P, Sabel-Goedknegt EH, Ruigrok TJ. Organization of cerebral projections to identified cerebellar zones in the posterior cerebellum of the rat. J Neurosci. 2012;32(32):10854-69.

9. Wickersham IR, Lyon DC, Barnard RJ, Mori T, Finke S, Conzelmann KK, et al. Monosynaptic restriction of transsynaptic tracing from single, genetically targeted neurons. Neuron. 2007;53(5):639-47.

10. Wall NR, Wickersham IR, Cetin A, De La Parra M, Callaway EM. Monosynaptic circuit tracing in vivo through Cre-dependent targeting and complementation of modified rabies virus. Proc Natl Acad Sci U S A. 2010;107(50):21848-53.

11. Tovote P, Esposito MS, Botta P, Chaudun F, Fadok JP, Markovic M, et al. Midbrain circuits for defensive behaviour. Nature. 2016;534(7606):206-12.

12. Foust KD, Nurre E, Montgomery CL, Hernandez A, Chan CM, Kaspar BK. Intravascular AAV9 preferentially targets neonatal neurons and adult astrocytes. Nat Biotechnol. 2009;27(1):59-65.

13. Foust KD, Wang X, McGovern VL, Braun L, Bevan AK, Haidet AM, et al. Rescue of the spinal muscular atrophy phenotype in a mouse model by early postnatal delivery of SMN. Nat Biotechnol. 2010;28(3):271-4.

14. Saraiva J, Nobre RJ, Pereira de Almeida L. Gene therapy for the CNS using AAVs: the impact of systemic delivery by AAV9. J Control Release. 2016;241:94-109

15. Deverman BE, Pravdo PL, Simpson BP, Kumar SR, Chan KY, Banerjee A, et al. Cre-dependent selection yields AAV variants for widespread gene transfer to the adult brain. Nat Biotechnol. 2016;34(2):204-9.

16. Chan KY, Jang MJ, Yoo BB, Greenbaum A, Ravi N, Wu WL, et al. Engineered AAVs for efficient noninvasive gene delivery to the central and peripheral nervous systems. Nat Neurosci. 2017;20(8):1172-9.

17. Montardy Q, Zhou Z, Lei Z, Liu X, Zeng P, Chen C, et al. Characterization of glutamatergic VTA neural population responses to aversive and rewarding conditioning in freely-moving mice. Sci Bull. 2019;64(16):1167-78.

18. Yim CY, Mogenson GJ. Effect of picrotoxin and nipecotic acid on inhibitory response of dopaminergic neurons in the ventral tegmental area to stimulation of the nucleus accumbens. Brain Res. 1980;199(2):466-73.

19. Gysling K, Wang RY. Morphine-induced activation of A10 dopamine neurons in the rat. Brain Res. 1983;277(1):119-27.

20. Grace AA, Onn SP. Morphology and electrophysiological properties of immunocytochemically identified rat dopamine neurons recorded in vitro. J Neurosci. 1989;9(10):3463-81.

21. Johnson SW, North RA. Opioids excite dopamine neurons by hyperpolarization of local interneurons. J Neurosci. 1992;12(2):483-8.

22. Sesack SR, Grace AA. Cortico-Basal Ganglia reward network: microcircuitry. Neuropsychopharmacology. 2010;35(1):27-47.

23. Bouarab C, Thompson B, Polter AM. VTA GABA neurons at the interface of stress and reward. Front Neural Circuits. 2019:13:78.
24. Li SJ, Vaughan A, Sturgill JF, Kepecs A. A viral receptor complementation strategy to overcome CAV-2 tropism for efficient retrograde targeting of neurons. Neuron. 2018:98(5):905-917 e5.

25. Sano H, Kobayashi K, Yoshioka N, Takebayashi H, Nambu A. Retrograde gene transfer into neural pathways mediated by adeno-associated virus (AAV)-AAV receptor interaction. J Neurosci Methods. 2020;345: 108887.

26. Sun L, Tang Y, Yan K, Yu J, Zou Y, Xu W, et al. Differences in neurotropism and neurotoxicity among retrograde viral tracers. Mol Neurodegener. 2019:14(1):8.

27. Miyamichi K, Shlomai-Fuchs Y, Shu M, Weissbourd BC, Luo L, Mizrahi A. Dissecting local circuits: parvalbumin interneurons underlie broad feedback control of olfactory bulb output. Neuron. 2013;80(5):1232-45.

28. Seidler B, Schmidt A, Mayr U, Nakhai H, Schmid RM, Schneider G, et al. A Cre-loxP-based mouse model for conditional somatic gene expression and knockdown in vivo by using avian retroviral vectors. Proc Natl Acad Sci U S A. 2008;105(29):10137-42.

29. Callaway EM, Luo L. Monosynaptic circuit tracing with glycoproteindeleted rabies viruses. J Neurosci. 2015;35(24):8979-85.

30. Grove J, Marsh M. The cell biology of receptor-mediated virus entry. J Cell Biol. 2011:195(7):1071-82.

31. Schneider-Schaulies J. Cellular receptors for viruses: links to tropism and pathogenesis. J Gen Virol. 2000;81 (Pt 6):1413-29.

32. Kato S, Kobayashi K, Inoue K, Kuramochi M, Okada T, Yaginuma H, et al. A lentiviral strategy for highly efficient retrograde gene transfer by pseudotyping with fusion envelope glycoprotein. Hum Gene Therapy. 2011;22(2):197-206.

33. Kato S, Kobayashi K, Inoue K, Takada M, Kobayashi K. Vectors for highly efficient and neuron-specific retrograde gene transfer for gene therapy of neurological diseases.In: In: Martin DF, editor. Gene Therapy: Tools and Potential Applications. Haverhill, MA. In Tech; 2013. p. 387-98.

34. Kobayashi K, Inoue KI, Tanabe S, Kato S, Takada M, Kobayashi K. Pseudotyped lentiviral vectors for retrograde gene delivery into target brain regions. Front Neuroanat. 2017;11:65.

35. Tanabe S, Inoue KI, Tsuge H, Uezono S, Nagaya K, Fujiwara M, et al. The use of an optimized chimeric envelope glycoprotein enhances the efficiency of retrograde gene transfer of a pseudotyped lentiviral vector in the primate brain. Neurosci Res. 2017;120:45-52.

36. Soudais C, Laplace-Builhe C, Kissa K, Kremer EJ. Preferential transduction of neurons by canine adenovirus vectors and their efficient retrograde transport in vivo. FASEB J. 2001;15(10):2283.

37. Ugolini $G$, Kuypers H, Simmons A. Retrograde trans-neuronal transfer of herpes-simplex virus type-1 (HSV 1) from motoneurons. Brain Res. 1987:422(2):242-56

38. Salegio EA, Samaranch L, Kells AP, Mittermeyer G, San Sebastian W, Zhou $\mathrm{S}$, et al. Axonal transport of adeno-associated viral vectors is serotypedependent. Gene Ther. 2013;20(3):348-52.

39. San Sebastian W, Samaranch L, Heller G, Kells AP, Bringas J, Pivirotto P, et al. Adeno-associated virus type 6 is retrogradely transported in the non-human primate brain. Gene Ther. 2013;20(12):1178-83.

40. Davidsson M, Wang G, Aldrin-Kirk P, Cardoso T, Nolbrant S, Hartnor M, et al. A systematic capsid evolution approach performed in vivo for the design of AAV vectors with tailored properties and tropism. Proc Natl Acad Sci USA. 2019:116(52):27053-62.

41. Root DH, Mejias-Aponte CA, Zhang S, Wang HL, Hoffman AF, Lupica CR, et al. Single rodent mesohabenular axons release glutamate and GABA. Nat Neurosci. 2014;17(11):1543-51.

42. Nieh EH, Matthews GA, Allsop SA, Presbrey KN, Leppla CA, Wichmann R, et al. Decoding neural circuits that control compulsive sucrose seeking. Cell. 2015;160(3):528-41.

43. de Git KCG, Hazelhoff EM, Nota MHC, Schele E, Luijendijk MCM, Dickson $S L$, et al. Zona incerta neurons projecting to the ventral tegmental area promote action initiation towards feeding. J Physiol. 2021;599(2):709-24.

44. Hernandez NS, Weir VR, Ragnini K, Merkel R, Zhang Y, Mace K, et al. GLP-1 receptor signaling in the laterodorsal tegmental nucleus attenuates cocaine seeking by activating GABAergic circuits that project to the VTA. Mol Psychiatry. 2021;26(8):4394-408.

45. Matsuzaki Y, Konno A, Mochizuki R, Shinohara Y, Nitta K, Okada Y, et al. Intravenous administration of the adeno-associated virus-PHP.B capsid fails to upregulate transduction efficiency in the marmoset brain. Neurosci Lett. 2018;665:182-8. 
46. Hordeaux J, Wang Q, Katz N, Buza EL, Bell P, Wilson JM. The neurotropic properties of AAV-PHP.B are limited to C57BL/6J mice. Mol Therapy. 2018;26(3):664-8.

47. Chen YH, Keiser MS, Davidson BL. Adeno-associated virus production, purification, and titering. Curr Protoc Mouse Biol. 2018;8(4): e56.

48. Osakada F, Mori T, Cetin AH, Marshel JH, Virgen B, Callaway EM. New rabies virus variants for monitoring and manipulating activity and gene expression in defined neural circuits. Neuron. 2011;71(4):617-31.

49. Paxinos $G$, Franklin K. Paxinos and Franklin's the mouse brain in stereotaxic coordinates. Amsterdam: Elsevier/Academic Press; 2012.

\section{Publisher's Note}

Springer Nature remains neutral with regard to jurisdictional claims in published maps and institutional affiliations.

- fast, convenient online submission

- thorough peer review by experienced researchers in your field

- rapid publication on acceptance

- support for research data, including large and complex data types

- gold Open Access which fosters wider collaboration and increased citations

- maximum visibility for your research: over $100 \mathrm{M}$ website views per year

At BMC, research is always in progress.

Learn more biomedcentral.com/submissions 